\title{
On the 2-Systole of Stretched Enough Positive Scalar Curvature Metrics on $\mathbb{S}^{2} \times \mathbb{S}^{2}$
}

\author{
Thomas RICHARD †
}

$\dagger$ Univ Paris Est Creteil, CNRS, LAMA, F-94010 Creteil, France

E-mail: thomas.richard@u-pec.fr

URL: https://perso.math.u-pem.fr/richard.thomas/

$\ddagger$ Univ Gustave Eiffel, LAMA, F-77447 Marne-la-Vallée, France

Received July 07, 2020, in final form December 14, 2020; Published online December 17, 2020

https://doi.org/10.3842/SIGMA.2020.136

\begin{abstract}
We use recent developments by Gromov and Zhu to derive an upper bound for the 2-systole of the homology class of $\mathbb{S}^{2} \times\{*\}$ in a $\mathbb{S}^{2} \times \mathbb{S}^{2}$ with a positive scalar curvature metric such that the set of surfaces homologous to $\mathbb{S}^{2} \times\{*\}$ is wide enough in some sense.
\end{abstract}

Key words: scalar curvature; higher systoles; geometric inequalities

2020 Mathematics Subject Classification: 53C42; 53C20

\section{Introduction}

Recall that the systole of a compact Riemannian manifold $\left(M^{n}, g\right)$ is the length of the shortest non contractible loop in $\left(M^{n}, g\right)$. In the middle of the 20th century, Loewner and Pu proved sharp upper bounds on the systole of any metric on $\mathbb{T}^{2}$ or $\mathbb{R} \mathbb{P}^{2}$ in term of its volume. These were vastly generalized when in the early 80s Gromov gave similar (non sharp) bounds for $n$-dimensional essential manifolds. (See [1] for the full story until 1993.)

The $k$-systole $\operatorname{sys}_{k}(g)$ of $\left(M^{n}, g\right)$ is the infimum of the $k$-dimensional volume over all homologically non trivial $k$-cycles. In general, for $k \geq 2$, the $k$-systole of a manifold cannot be bounded by the volume alone. In particular, Katz and Suciu showed in [8] that one can find metrics on $\mathbb{S}^{2} \times \mathbb{S}^{2}$ whose volume is 1 but whose 2 -systole can be arbitrary large. (Similar examples in higher dimension where already known to Gromov, see again [1].)

One way to circumvent this is to introduce the more subtle "stable systoles" (see again [1]), another way is to try to introduce curvature restrictions. This second route was first considered in dimension 3 by Bray, Brendle and Neves for manifolds such as $\mathbb{S}^{2} \times \mathbb{S}^{1}$ with positive scalar curvature metrics in [3]. Recently, Zhu treated the case of $\mathbb{S}^{2} \times \mathbb{T}^{n}(n+2 \leq 7)$ in [9], see Section 2 for a precise statement.

Here we will show how Zhu's result together with recent developments due to Gromov gives some progress in the case of $\mathbb{S}^{2} \times \mathbb{S}^{2}$.

Let $\mathcal{S}_{\ell}$ be the set of embedded surfaces in $\mathbb{S}^{2} \times \mathbb{S}^{2}$ which are in the same homology class as $\mathbb{S}^{2} \times\{*\}$.

Definition 1.1. Let $g$ be a Riemannian metric on $\mathbb{S}^{2} \times \mathbb{S}^{2}$, the left stretch of $g$, denoted by $\operatorname{stretch}_{\ell}(g)$ is defined as

$$
\operatorname{stretch}_{\ell}(g)=\sup _{S_{1}, S_{2} \in \mathcal{S}_{\ell}} d_{g}\left(S_{1}, S_{2}\right) .
$$

This paper is a contribution to the Special Issue on Scalar and Ricci Curvature in honor of Misha Gromov on his 75th Birthday. The full collection is available at https://www.emis.de/journals/SIGMA/Gromov.html 
Example 1.2. For a product metric $g=g_{1} \oplus g_{2}$ on $\mathbb{S}^{2} \times \mathbb{S}^{2}$, the left stretch is $\operatorname{stretch}_{\ell}(g)=$ $\operatorname{diam}\left(\mathbb{S}^{2}, g_{2}\right)$ and is achieved by $S_{1}=\mathbb{S}^{2} \times\left\{p_{1}\right\}$ and $S_{2}=\mathbb{S}^{2} \times\left\{p_{2}\right\}$ where $d_{g_{2}}\left(p_{1}, p_{2}\right)=$ $\operatorname{diam}\left(\mathbb{S}^{2}, g_{2}\right)$.

As the example above shows, the left stretch is a measure of the 1-dimensional size of the right factor of $\left(\mathbb{S}^{2} \times \mathbb{S}^{2}, g\right)$. It is bounded above by the diameter of $g$.

Definition 1.3. Let $g$ be a Riemannian metric on $\mathbb{S}^{2} \times \mathbb{S}^{2}$, the left 2-systole of $g$, denoted by $\operatorname{sys}_{2, \ell}(g)$ is defined as

$$
\operatorname{sys}_{2, \ell}(g)=\inf _{S \in \mathcal{S}_{\ell}} \operatorname{area}_{g}(S)
$$

Example 1.4. For a product metric $g=g_{1} \oplus g_{2}$ on $\mathbb{S}^{2} \times \mathbb{S}^{2}$, the left 2 -systole is $\operatorname{sys}_{2, \ell}(g)=$ area $\left(\mathbb{S}^{2}, g_{1}\right)$ and is achieved by any $S=\mathbb{S}^{2} \times\{*\}$.

The theorem below is the main result of the paper. It says that positive scalar curvature metrics on $\mathbb{S}^{2} \times \mathbb{S}^{2}$ with large left stretch cannot have large left 2-systole.

Theorem 1.5. Let $g$ be a metric on $\mathbb{S}^{2} \times \mathbb{S}^{2}$ with $\mathrm{Scal}_{g} \geq 4$.

If $s=\operatorname{stretch}_{\ell}\left(\mathbb{S}^{2} \times \mathbb{S}^{2}, g\right)>\frac{\sqrt{3} \pi}{2}$, then $\operatorname{sys}_{\ell}\left(\mathbb{S}^{2} \times \mathbb{S}^{2}, g\right) \leq \frac{8 \pi s^{2}}{4 s^{2}-3 \pi^{2}}$. Moreover there is an embedded 2-sphere whose area is at most $\frac{8 \pi s^{2}}{4 s^{2}-3 \pi^{2}}$.

Remark 1.6. This estimate is asymptotically sharp when $s$ goes to $+\infty$, as the example of the product fo two round spheres of radi $\frac{1}{\sqrt{k}}$ and $\frac{1}{\sqrt{2-k}}$ for $k \in[1,2)$ shows. It remains unclear to the author wether this estimate is optimal or not. What can be shown from tracking down the equality case in the proofs of Theorems 2.1 and 2.4 is that if the inequality in Theorem 1.5 is an equality then $M$ contains an hypersurface $\Sigma$ such that:

- There exists a map $\Sigma \rightarrow \mathbb{S}^{2} \times \mathbb{S}^{1}$ with non-vanishing degre.

- The universal cover of $\Sigma$ is isometric to $\mathbb{S}^{2} \times \mathbb{R}$ with the product metric such that the $\mathbb{S}^{2}$ factor is round and has area exactly $\frac{8 \pi s^{2}}{4 s^{2}-3 \pi^{2}}$.

Products of two round $\mathbb{S}^{2}$ which satisfy the properties above have left stretch smaller than $s$. The investigation of doubly warped product metrics of the form $\psi^{2}(r) g_{\mathbb{S}^{2}}+\varphi^{2}(r) \mathrm{d} \theta^{2}+\mathrm{d} r^{2}$ on $\mathbb{S}^{2} \times \mathbb{S}^{2}$ was inconclusive too.

Remark 1.7. Of course, if one denotes by $\mathcal{S}_{r}$ the set of embedded spheres homologous to $\{*\} \times \mathbb{S}^{2}$, we can define right stretch and systole as $\operatorname{stretch}_{r}(g)=\sup _{S_{1}, S_{2} \in \mathcal{S}_{r}} d_{g}\left(S_{1}, S_{2}\right)$ and $\operatorname{sys}_{2, r}(g)$ $=\inf _{S \in \mathcal{S}_{r}} \operatorname{area}_{g}(S)$ and get the same inequality between the right systole and the right stretch.

It is currently unknown wether the 2 -systole is bounded from above on the set $\mathcal{M}_{\mathbb{S}^{2} \times \mathbb{S}^{2} \text {,Scal } \geq 4}$ of metrics on $\mathbb{S}^{2} \times \mathbb{S}^{2}$ with scalar curvature greater than 4 . Our result says that if there is no upper bound, there must be a sequence of metrics in $\mathcal{M}_{\mathbb{S}^{2} \times \mathbb{S}^{2}, \text { Scal } \geq 4}$ whose 2 -systoles goes to infinity while their left and right stretches stay below $\frac{\sqrt{3}}{2} \pi$.

The rest of the paper is organized as follows: we first review some previously known results by Gromov and Zhu on manifolds with positive scalar curvature which will be used to prove Theorem 1.5, we then prove some topological facts that will be needed in the proof of Theorem 1.5 and we finally give the proof of the main theorem. 


\section{Positive scalar curvature inequalities by Gromov and Zhu}

We will need the following result by Jintian Zhu, which was already alluded to in the introduction.

Theorem 2.1 ([9, Theorem 1.2]). Let $\left(M^{n}, g\right)$ be a closed manifold of dimension at most seven such that:

- $\mathrm{Scal}_{g} \geq 2$;

- there exists a map $F: M^{n} \rightarrow \mathbb{S}^{2} \times \mathbb{T}^{n-2}$ with non-vanishing degree.

Then $\operatorname{sys}_{2}(g) \leq 4 \pi$. More precisely one can find an embedded 2 -sphere $S$ such that $F_{*}([S])=$ $\left[\mathbb{S}^{2} \times\{*\}\right]$ and whose area is less than $4 \pi$.

The proof uses the second variation formula of the area functional for stable minimal hypersurfaces and a repeated symmetrization construction to reduce the problem to $\mathbb{T}^{n-2}$-invariant metrics on $\Sigma^{2} \times \mathbb{T}^{n-2}$. This method of proof was pioneered by Fischer-Colbrie and Schoen in dimension 3 and used in dimensions at most seven by Gromov and Lawson in [6]. Zhu also studies the equality case: under the hypothesis of the theorem, if there is an embedded 2-sphere $S$ such that $F_{*}([S])=\left[\mathbb{S}^{2} \times\{*\}\right]$ and whose area is $4 \pi$, then the universal cover of $M$ is a product of the round 2 -sphere of area $4 \pi$ and a euclidean space.

Remark 2.2. The proof in [9] shows that if one starts with an $\mathbb{S}^{1}$-invariant metric on $M=$ $M^{\prime} \times \mathbb{S}^{1}$ satisfying the hypothesis of Theorem 2.1, then one can find a 2-sphere in $M^{\prime}$ whose area is at most $4 \pi$.

In the Spring of 2019, the author was lucky enough to attend the lectures "Old, New and Unknown around Scalar Curvature" given by Gromov at IHES. There, the author learned about the following result by Gromov which is also proved using the Fischer-Colbrie-Schoen symmetrization process:

Theorem 2.3 ([4, p. 2]). Let $2 \leq n \leq 7, M=[-1,1] \times \mathbb{T}^{n-1}$ and $\partial_{ \pm} M=\{ \pm 1\} \times \mathbb{T}^{n-1}$. Let $g$ be a Riemannian metric on $M$ such that $\mathrm{Scal}_{g} \geq n(n-1)$. Then

$$
d_{g}\left(\partial_{-} M, \partial_{+} M\right) \leq \frac{2 \pi}{n} .
$$

The equality case is also studied. If $d_{g}\left(\partial_{-} M, \partial_{+} M\right)=\frac{2 \pi}{n}$, then $M$ is isometric to $\left[-\frac{\pi}{n}, \frac{\pi}{n}\right] \times$ $\mathbb{T}^{n-1}$ with the product metric $\mathrm{d} t^{2}+\cos ^{\frac{4}{n}}\left(\frac{n t}{2}\right) \mathrm{d} x^{2}$ where $\mathrm{d} x^{2}$ is a flat metric on $\mathbb{T}^{n-1}$.

In his Spring 2019 lectures, Gromov gave a new proof of the previous theorem. Instead of using the second variation of the $(n-1)$-dimensional volume as in [4], a twisted functional is considered. Given a density $\mu: M^{n} \rightarrow \mathbb{R}$, the $\mu$-area functional maps an open set $U \subset M$ to $\mathcal{V}_{n-1}(\partial U)-\int_{U} \mu$, where $\mathcal{V}_{n-1}$ denotes the $(n-1)$-dimensional volume. Using a well chosen density $\mu$, Gromov proved the following theorem, which is a generalisation of the theorem above.

Theorem 2.4 ([5, Section 5.3]). Let $\left(M^{n}, g\right)(n \leq 7)$ be a compact $n$-manifold with two boundary components $\partial_{-} M$ and $\partial_{+} M$. Assume that

$$
\mathrm{Scal}_{g} \geq \frac{4(n-1) \pi^{2}}{n d_{g}\left(\partial_{-} M, \partial_{+} M\right)^{2}}+\delta
$$

for some $\delta>0$. Then there exists

- an hypersurface $\Sigma$ which separates $\partial_{-} M$ and $\partial_{+} M$,

- a positive function $u: \Sigma \rightarrow \mathbb{R}$,

such that the metric $h=\left.g\right|_{\Sigma}+u^{2} \mathrm{~d} t^{2}$ on $\Sigma \times \mathbb{R}$ has $\mathrm{Scal}_{h} \geq \delta$. 


\section{$3 \quad$ Topological preliminaries}

Before proving the main theorem, we establish a topological preliminary. Let $M=\mathbb{S}^{2} \times \mathbb{S}^{2}$.

The idea is that if we remove two disjoint surfaces $S_{1}$ and $S_{2}$ in $\mathcal{S}_{\ell}$ from $M$, we should be in a situation which is very similar to removing $\mathbb{S}^{2} \times\{n\}$ and $\mathbb{S}^{2} \times\{s\}$ from $\mathbb{S}^{2} \times \mathbb{S}^{2}$, where $n$ and $s$ are the north and south poles of the right factor. Thus $\tilde{M}=M \backslash\left(S_{1} \cup S_{2}\right)$ should look like

$$
\mathbb{S}^{2} \times \mathbb{S}^{2} \backslash\left(\mathbb{S}^{2} \times\{n\} \cup \mathbb{S}^{2} \times\{s\}\right)=\mathbb{S}^{2} \times\left(\mathbb{S}^{2} \backslash\{n, s\}\right) \simeq \mathbb{S}^{2} \times \mathbb{S}^{1} \times(-1,1) .
$$

However, our surfaces $S_{1}$ and $S_{2}$ from $\mathcal{S}_{\ell}$ may have higher genus and thus $\tilde{M}$ may be more complicated.

However we are able to prove the following, which will be enough for our purpose:

Proposition 3.1. Let $S_{1}$ and $S_{2}$ be two disjoint surfaces in $\mathcal{S}_{\ell}$. Let $\Sigma \subset M$ be a connected hypersurface such that

- $\Sigma$ is disjoint from $S_{1}$ and $S_{2}$,

- $\Sigma$ separates $S_{1}$ and $S_{2}$.

Then there exists a non-zero degree map $\Sigma \rightarrow \mathbb{S}^{2} \times \mathbb{S}^{1}$.

This map will be the restriction of a map $F: \tilde{M}=M \backslash\left(S_{1} \cup S_{2}\right) \rightarrow \mathbb{S}^{2} \times \mathbb{S}^{1}$.

We will first show:

Lemma 3.2. Any $S \in \mathcal{S}_{\ell}$ has a trivial normal bundle.

Proof. Let $N S$ be the normal bundle to $S$ and let $e(N S)$ be the Euler class of $N S$. It follows from $[2$, Theorem 11.17 , p. 125] that the Euler class of a vector bundle over a compact manifold can be computed as the intersection number between the zero section and another transverse section times the fundamental class of the base.

Since $N S$ can be embedded in $M$ as a small tubular neighborhood of $N$, this intersection number can be computed in $M$. Since in $\mathcal{S}_{\ell}$ one can find two disjoint spheres, the intersection number is 0 and $e(N S)=0$.

Moreover the Euler class is the only obstruction for an oriented rank $k$ vector bundle over a compact $k$-manifold to have a non vanishing section (see [7, Proposition 3.22]). Hence $N S$ has a nowhere vanishing section. Since $N S$ is an orientable rank 2 vector bundle, it is trivial.

For $\varepsilon>0$, we denote by $S_{i}^{\varepsilon}$ the tubular neighborhood around $S_{i}$. We will choose $\varepsilon$ small enough so that $S_{1}^{\varepsilon}$ and $S_{2}^{\varepsilon}$ are disjoint regular neighborhoods.

Lemma 3.3. The map $F_{2}: \tilde{M} \subset \mathbb{S}^{2} \times \mathbb{S}^{2} \rightarrow \mathbb{S}^{2} \times\{*\}$ is surjective in homology.

Proof. Consider a 3-cycle $C$ in $M$ such that $\partial C=S_{1}-S_{2}$. By using an fine enough triangulation, we can decompose $C=C_{1}+\tilde{C}+C_{2}$, where $\tilde{C}$ is a 3 -cycle in $\tilde{M}$ and $C_{i}$ is a 3 -cycle in $S_{i}^{\varepsilon}$. Now, $S_{1}^{\prime}=\partial C_{1}-S_{1}$ is a 2 -cycle in $\tilde{M}$ homologous to $S_{1}$, hence $\pi\left(F_{2}\right)_{*}\left(\left[S_{1}^{\prime}\right]\right)=\left[\mathbb{S}^{2} \times\{*\}\right]$.

Set $S=S_{1} \cup S_{2}, S^{\varepsilon}=S_{1}^{\varepsilon} \cup S_{2}^{\varepsilon}$ and $\tilde{S}^{\varepsilon}=S^{\varepsilon} \backslash S$.

Lemma 3.4. $H^{1}(\tilde{M}, \mathbb{Z})=\mathbb{Z}$.

Proof. We will use the Mayer-Vietoris exact sequence for cohomology associated with the decomposition $M=S^{\varepsilon} \cup \tilde{M}$. Note that $S^{\varepsilon}$ is homotopy equivalent to $S$ and since $S_{1}$ and $S_{2}$ have trivial normal bundle, $\tilde{S}^{\varepsilon}$ is homotopy equivalent to $S \times \mathbb{S}^{1}$.

The Mayer-Vietoris sequence gives

$$
\cdots \rightarrow H^{1}(M) \rightarrow H^{1}\left(S^{\varepsilon}\right) \oplus H^{1}(\tilde{M}) \rightarrow H^{1}\left(\tilde{S}^{\varepsilon}\right) \rightarrow H^{2}(M) \rightarrow \cdots .
$$


Thus, we have

$$
\cdots \rightarrow 0 \rightarrow H^{1}\left(S_{1}\right) \oplus H^{1}\left(S_{2}\right) \oplus H^{1}(\tilde{M}) \rightarrow H^{1}\left(S_{1}\right) \oplus \mathbb{Z} \oplus H^{1}\left(S_{2}\right) \oplus \mathbb{Z} \rightarrow \mathbb{Z} \oplus \mathbb{Z} \rightarrow \cdots,
$$

where the map $H^{1}\left(\tilde{S}^{\varepsilon}\right) \simeq H^{1}\left(S_{1}\right) \oplus \mathbb{Z} \oplus H^{1}\left(S_{2}\right) \oplus \mathbb{Z} \rightarrow H^{2}(M)$ is given by $(a, x, b, y) \mapsto(x-y, 0)$. Hence $H^{1}(\tilde{M})$ is isomorphic to $\mathbb{Z}$.

Since $H^{1}(\tilde{M}, \mathbb{Z}) \simeq \mathbb{Z}, H^{1}(\tilde{M}, \mathbb{R}) \simeq \mathbb{R}$ and we can find a closed 1-form $\alpha$ on $\tilde{M}$ which is not exact. Let $\Gamma$ be the image in $\mathbb{R}$ of the abelian group morphism: $c \in H_{1}(\tilde{M}, \mathbb{Z}) \mapsto \int_{c} \alpha \in \mathbb{R}$. By a computation similar to the proof of the previous lemma, $H_{1}(\tilde{M}, \mathbb{Z})=\mathbb{Z}$. Hence $\Gamma$ is a discrete subgroup of $\mathbb{R}$. The classical period map construction gives:

Lemma 3.5. Let $x_{0} \in \tilde{M}$. For any curve $c$ from $x_{0}$ to $x, \int_{c} \alpha$ is well defined as an element $\mathbb{R} / \Gamma$. This defines a map $F_{1}: \tilde{M} \rightarrow \mathbb{R} / \Gamma$ such that the induced map $H_{1}(\tilde{M}) \rightarrow H_{1}(\mathbb{R} / \Gamma)$ is an isomorphism.

$$
F=\left(F_{2}, F_{1}\right): \tilde{M} \rightarrow \mathbb{S}^{2} \times \mathbb{S}^{1} \text { is the map we are looking for. }
$$

Proof of Proposition 3.1. First note that any connected hypersurface $\Sigma \subset M$ which separates $S_{1}$ and $S_{2}$ will be homologous to $\partial S_{1}^{\varepsilon}$ for $\varepsilon$ small enough. Thus it is enough to show that the image of $\left[\partial S_{1}^{\varepsilon}\right]$ under $F$ is not zero in $H_{3}\left(\mathbb{S}^{2} \times \mathbb{S}^{1}\right)=H_{2}\left(\mathbb{S}^{2}\right) \oplus H_{1}\left(\mathbb{S}^{1}\right)$, which is routinely deduced from the proofs of Lemmas 3.3 and 3.4.

We will also need an elementary fact about the connectedness of separating hypersurfaces. Before stating it let us recall that $\Sigma \subset M$ separates $K_{1}, K_{2} \subset M$ if $K_{1}$ and $K_{2}$ lie in different connected components of $M \backslash \Sigma$. The result we will need is the following:

Proposition 3.6. Let $M^{n}$ be a closed orientable manifold with $H_{n-1}(M)=0$ and let $\Sigma^{n-1} \subset M^{n}$ be a closed orientable hypersurface. Let $K_{1}$ and $K_{2}$ be two closed subsets of $M$ such that $\Sigma$ separates $K_{1}$ and $K_{2}$. Then there is a connected component of $\Sigma$ which separates $K_{1}$ and $K_{2}$.

This is probably well known but the author has not been able to locate a proof in the litterature.

Proof. Let us first remark that since $H_{n-1}(M)=0$, any connected closed hypersurface $\Sigma_{0} \subset M$ satisfies that $M \backslash \Sigma_{0}$ has exactly two connected components whose boundary in $M$ is $\Sigma_{0}$. The fact that $M \backslash \Sigma_{0}$ has at most two connected components follows from the fact $\Sigma_{0}$ is two sided (since both $\Sigma_{0}$ and $M$ are orientable). If $M \backslash \Sigma_{0}$ was connected, there would be a closed loop $\gamma$ which intersects $\Sigma_{0}$ exactly once, hence the intersection number of $[\gamma] \in H_{1}(M)$ and $\left[\Sigma_{0}\right] \in H_{n-1}(M)$ would be non zero, which is absurd since $H_{n-1}(M)=0$.

We will now prove the following result: let $\tilde{\Sigma}$ and $\Sigma_{0}$ be two disjoint hypersurfaces such that:

- $\Sigma_{0}$ is connected.

- $\tilde{\Sigma} \cup \Sigma_{0}$ separates $K_{1}$ and $K_{2}$.

- $\Sigma_{0}$ does not separate $K_{1}$ and $K_{2}$.

Then $\tilde{\Sigma}$ separates $K_{1}$ and $K_{2}$.

Once we have proved this, the proposition follows since we can remove all the non-separating components form $\Sigma$ until every connected component of $\Sigma$ separates $K_{1}$ and $K_{2}$.

In order to prove the result above, let $U_{0}$ be the connected component of $M \backslash \Sigma_{0}$ which contains $K_{1}$. Since $\Sigma_{0}$ does not separate $K_{1}$ and $K_{2}, U_{0}$ also contains $K_{2}$. Now let $\tilde{U}$ be the connected component of $M \backslash \tilde{\Sigma}$ which contains $K_{1}$. 
Since the boundaries of $U_{0}$ and $\tilde{U}$ are disjoint and both $U_{0}$ and $\tilde{U}$ contain $K_{1}$ either $U_{0} \subset \tilde{U}$ or $\tilde{U} \subset U_{0}$. In the first case, $U_{0}$ will be a connected component of $M \backslash \Sigma$ which contains $K_{1}$ and $K_{2}$, which is impossible since $\Sigma$ separates $K_{1}$ and $K_{2}$.

Hence $\tilde{U} \subset U_{0}$, which implies that $\tilde{U}$ is one of the connected components of $M \backslash \Sigma$. Since $\Sigma$ separates $K_{1}$ and $K_{2}, K_{2}$ cannot be included in $\tilde{U}$. Hence $\tilde{\Sigma}$ separates $K_{1}$ and $K_{2}$.

\section{Proof of the main theorem}

We are now ready to prove Theorem 1.5.

Proof of Theorem 1.5. Our assumption is that $s=\operatorname{stretch}_{\ell}\left(\mathbb{S}^{2} \times \mathbb{S}^{2}, g\right)>\frac{\sqrt{3} \pi}{2}$. Thus, for any $\varepsilon>0$ we can find two surfaces $S_{1}$ and $S_{2}$ in $\mathcal{S}_{\ell}$ such that $d_{g}\left(S_{1}, S_{2}\right)=s-\varepsilon$. Assume that $\varepsilon>0$ is such that

- $s-3 \varepsilon>\frac{\sqrt{3} \pi}{2}$;

- the tubular neighborhoods $S_{1}^{\varepsilon}$ and $S_{2}^{\varepsilon}$ of radius $\varepsilon$ around $S_{1}$ and $S_{2}$ are disjoint and regular.

Then if we set $\tilde{M}=M \backslash\left(S_{1}^{\varepsilon} \cup S_{2}^{\varepsilon}\right)$, its two boundary components $\partial_{-} \tilde{M}=\partial S_{1}^{\varepsilon}$ and $\partial_{+} \tilde{M}=\partial S_{2}^{\varepsilon}$ satisfy $d_{g}\left(\partial_{-} \tilde{M}, \partial_{+} \tilde{M}\right)=s-3 \varepsilon$.

Set $\delta=4-\frac{3 \pi^{2}}{(s-3 \varepsilon)^{2}}$ and note that $\delta>0$. Then we have

$$
\mathrm{Scal}_{g} \geq 4=\frac{3 \pi^{2}}{d_{g}\left(\partial_{-} \tilde{M}, \partial_{+} \tilde{M}\right)^{2}}+\delta .
$$

This is exactly what we need to apply Gromov's Theorem 2.4. Hence we get an hypersurface $\Sigma \subset \tilde{M}$ which separates $S_{1}$ and $S_{2}$ such that $\Sigma \times \mathbb{S}^{1}$ admits a metric of the form $h=g_{\Sigma}+u^{2} \mathrm{~d} t^{2}$ for some $u: M \rightarrow \mathbb{R}$ such that $\mathrm{Scal}_{h} \geq \delta$. The hypersurface coming the minimization process in the proof of Theorem 2.4 may be disconnected, however by Proposition 3.6 we can replace $\Sigma$ by one of its connected components which separates $S_{1}$ and $S_{2}$.

Moreover, by Proposition 3.1, there exists a non-zero degree map $\Sigma \rightarrow \mathbb{S}^{2} \times \mathbb{S}^{1}$. Hence there is a non-zero degree map $\Sigma \times \mathbb{S}^{1} \rightarrow \mathbb{S}^{2} \times \mathbb{T}^{2}$ and we can apply Zhu's Theorem 2.1 and Remark 2.2 to show that one can find an embedded 2-sphere $S$ in $\Sigma$ whose area is at most $\frac{8 \pi}{\delta}=\frac{8 \pi(s-3 \varepsilon)^{2}}{4(s-3 \varepsilon)^{2}-3 \pi^{2}}$.

Since $\Sigma$ is isometrically embedded in $M, S$ embeds in $M$ with area at most $\frac{8 \pi(s-3 \varepsilon)^{2}}{4(s-3 \varepsilon)^{2}-3 \pi^{2}}$. Moreover, by the properties of the map $F$ built in Section 3, the surface $S$ of $M$ belongs to $\mathcal{S}_{\ell}$. Since $\varepsilon>0$ can be as small as one wants we get the results.

\section{Acknowledgements}

The author would like to thank G. Besson, S. Maillot and S. Sabourau for helpful discussions. He also thanks Jintian Zhu and the anonymous referees for pointing inaccuracies in a previous version of this work. The author is supported by the grant ANR-17-CE40-0034 of the French National Research Agency ANR (Project CCEM).

\section{References}

[1] Berger M., Systoles et applications selon Gromov, Astérisque 216 (1993), 771, 279-310.

[2] Bott R., Tu L.W., Differential forms in algebraic topology, Graduate Texts in Mathematics, Vol. 82, SpringerVerlag, New York - Berlin, 1982.

[3] Bray H., Brendle S., Neves A., Rigidity of area-minimizing two-spheres in three-manifolds, Comm. Anal. Geom. 18 (2010), 821-830, arXiv:1002.2814. 
[4] Gromov M., Metric inequalities with scalar curvature, Geom. Funct. Anal. 28 (2018), 645-726, arXiv:1710.04655.

[5] Gromov M., Four lectures on scalar curvature, arXiv:1908.10612.

[6] Gromov M., Lawson Jr. H.B., Positive scalar curvature and the Dirac operator on complete Riemannian manifolds, Inst. Hautes Études Sci. Publ. Math. 58 (1983), 83-196.

[7] Hatcher A., Vector bundles and K-theory, available at http://pi.math.cornell.edu/ hatcher/.

[8] Katz M.G., Suciu A.I., Volume of Riemannian manifolds, geometric inequalities, and homotopy theory, in Tel Aviv Topology Conference: Rothenberg Festschrift (1998), Contemp. Math., Vol. 231, Amer. Math. Soc., Providence, RI, 1999, 113-136, arXiv:math.DG/9810172.

[9] Zhu J., Rigidity of area-minimizing 2-spheres in $n$-manifolds with positive scalar curvature, Proc. Amer. Math. Soc. 148 (2020), 3479-3489, arXiv:1903.05785. 